\title{
Non-Archimedean character of quantum buoyancy and the generalized second law of thermodynamics
}

\author{
Jacob D. Bekenstein* \\ The Racah Institute of Physics, Hebrew University of Jerusalem, \\ Givat Ram, Jerusalem 91904, Israel
}

(June 7, 2021)

\begin{abstract}
Quantum buoyancy has been proposed as the mechanism protecting the generalized second law when an entropy-bearing object is slowly lowered towards a black hole and then dropped in. We point out that the original derivation of the buoyant force from a fluid picture of the acceleration radiation is invalid unless the object is almost at the horizon, because otherwise typical wavelengths in the radiation are larger than the object. The buoyant force is here calculated from the diffractive scattering of waves off the object, and found to be weaker than in the original theory. As a consequence, the argument justifying the generalized second law from buoyancy cannot be completed unless the optimal drop point is next to the horizon. The universal bound on entropy is always a sufficient condition for operation of the generalized second law, and can be derived from that law when the optimal drop point is close to the horizon. We also compute the quantum buoyancy of an elementary charged particle; it turns out to be negligible for energetic considerations. Finally, we speculate on the significance of the absence from the bound of any mention of the number of particle species in nature.
\end{abstract}

\section{INTRODUCTION}

An observer accelerating in flat and empty spacetime with acceleration $a$ detects isotropic thermal radiation with temperature $T_{U}=\hbar a / 2 \pi$, the celebrated Unruh radiance [1]. An object suspended in the vicinity of a black hole is accelerated by virtue of its being prevented from following a geodesic. Unruh and Wald (UW) [2] suggested that this object will likewise see Unruh radiance. Since its acceleration (hence $T_{U}$ ) varies with distance from the horizon, UW surmised that the object will be subject to a buoyant force, just as an object submerged in fluid in a gravitational field is buoyed up by the non-uniformity of the ambient pressure. Two intriguing consequences were inferred: the buoyancy can cause an object sufficiently near the horizon to "float", and the buoyancy affects the energetics of a process whereby an object is lowered from afar towards a black hole while doing work on the lowering mechanism [2].

\footnotetext{
*e-mail: bekenste@vms.huji.ac.il
} 
Both these effects bear on the important issue of what is the mechanism enforcing the generalized second law (GSL) [3] when an entropy-bearing object is lowered slowly towards a black hole, and then dropped in ? Our initial inquiry [4], carried out before the quantum buoyancy was put in evidence, assumed it is possible to lower the object almost to the horizon, and inferred from the GSL that its entropy, $S$, must be bounded in terms of its radius $R$ and mass-energy $E$ (units with $c=1$ throughout) by

$$
S \leq 2 \pi R E / \hbar
$$

This is to be regarded as a universal bound on the entropy of matter (radiation) calculated to the deepest level of structure. Bound (1) is now backed by much independent evidence from flat space physics (for a review see reference [5]), and was an antecedent of 't Hooft's and Susskind's holographic principle [6.]7, which can be deduced from the bound for weakly gravitating systems [8,9].

UW questioned the black hole derivation of bound (1) on the grounds that the quantum buoyancy allows free lowering of the object only down to the floating point, and modifies the naive energetics of the problem. They developed a three-steps argument [2] suggesting

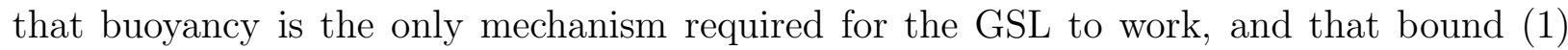
need not be invoked (and cannot thus be derived from the GSL). The steps are:

- 1. Calculation of the full energy of the object at the floating point, an energy which determines the minimum increase in black hole entropy, $\Delta S_{B H}$, occasioned when the object is dropped into the hole. UW claim that $\Delta S_{B H}$ is never smaller than $\mathcal{S}_{\text {disp }}$, the entropy in the Unruh radiance displaced by the object at the floating point.

- 2. Demonstration that Archimedes' principle is obeyed: at the floating point the energy of the displaced radiation equals the object's proper energy.

- 3. Proposition that the object's entropy $S$ cannot possibly exceed $\mathcal{S}_{\text {disp }}$ because unconfined thermal radiation is maximally entropic for given volume and energy (UW's entropy bound).

Combination of the three steps gives $\Delta S_{B H}>S$ : the GSL works because the black hole entropy overcompensates for the loss of the object's entropy. Other arguments that quantum buoyancy is sufficient by itself to protect the GSL have been offered by Li and Liu [10], Zaslavskii [11] and Pelath and Wald (PW) [12].

Following UW's original paper we noted that, provided the object is macroscopic (technically $\hbar / E \ll R$ ), and that the number $N$ of species present in the Unruh radiance is moderate, the floating point is necessarily very near the horizon [13] 15]. As a consequence the lowering process is almost unchanged by quantum buoyancy, with buoyant corrections to its energetics showing up late in the lowering process. This has allowed bound (1) to be rederived from the GSL applied to the described gedanken experiment even in the face of buoyancy [10,15,11].

PW [12] have kindled the controversy anew by asserting that this feat is made possible only because the same assumptions whose outcome is to put the floating point very near the horizon will cause bound (11) to be satisfied automatically without any recourse to black hole physics. PW base their argument on UW's bound on entropy (step 3 above). Exceptions 
to the UW's bound have been demonstrated in flat spacetime [14]: an object's entropy can exceed that of an equal energy and volume of unconfined thermal radiation if one (or two) of the object's dimensions is (are) much larger than the third. In the present paper we mostly focus on a spherical object for which the UW bound may well hold; this allows us to shift attention to the first two steps of UW's argument.

So is bound (11) a condition for the GSL to work in accretion by a black hole ? Or is quantum buoyancy the sole protector of that law, so that the bound cannot be inferred from it ? Given the importance of the GSL as an extension of the very pervasive second law, and as giving operational meaning to the concept of black hole entropy, it is obviously important to elucidate the questions raised. In addition, one would like to clarify how allowing for the existence of an arbitrary number of species in the radiation bears on all earlier conclusions. Both UW and PW are of the opinion that the GSL should be shown to be upheld by a single mechanism for arbitrary $N$. The present paper provides substantial clarification of all these issues.

In Sec.II we point out that buoyancy away from the horizon has been calculated incorrectly in all extant works. Departures from the fluid description of the Unruh radiance result because wavelenghts in the radiance are typically longer than the object's size. Sec.[II deals with a spherical object always removed from the horizon by a proper distance larger than its own size. In Sec.IIIB we calculate the buoyancy on it, and find it to be weaker and more rapidly falling with distance from the horizon than would be expected from UW's approach. Sec.IIC we make it clear that Archimedes' principle fails in this far region, and also that UW's "GSL from buoyancy" argument cannot be completed. By contrast, the entropy bound (1) is found to be a sufficient condition for the GSL to function.

In Sec.[V we consider an object whose floating point is much closer to the horizon than its own size. This case is relevant for a macroscopic object and a moderate number of radiance species. UW's fluid estimate of the buoyancy force is correct in this near region; however, their energetics are slightly changed because the object has to pass through the far region on its way down. Overall the UW argument that the GSL is protected by the buoyancy is upheld in the near region. On the other hand, in spite of the buoyancy, the entropy bound (1) is derivable from the GSL. In Sec. $\mathrm{V}$ we treat the intermediate region where the object's proper distance from the horizon is comparable to its size, and confirm the conclusion that the argument for the GSL from buoyancy cannot be completed over a certain regime; the entropy bound remains a sufficient condition for the GSL to work.

In Sec.VI we consider buoyancy of an elementary charged particle, finding it to be negligible under all circumstances. This is important for one of the arguments supporting the uniformly spaced black hole area quantum spectrum [16] 18]

\section{FLUID VS WAVE PICTURE}

All mentioned works follow UW in assuming that the object is impervious to the radiation, and that the buoyant force, as measured at infinity, can be calculated by integrating the radiance pressure $p$ multiplied by the local redshift factor $\chi$ and by the inward normal to the surface all around the object's surface. UW [2] relied on thermodynamic description of the radiance as a fluid obeying $e+p-T s=0$, where $e$ denotes the proper energy density, 
$s$ the proper entropy density, and $T$ the local temperature. They took $T$ in the vicinity of the horizon to obey

$$
T=T_{0} \chi^{-1}
$$

where $T_{0}$ denotes the Hawking temperature. One can justify this form by noting it is a good approximation to the formal Unruh temperature corresponding to the acceleration felt by a point suspended at rest in a Schwarzschild metric at distances from the horizon small compared to the size of the black hole [15, 11].

While there is little doubt about the cogency of the thermodynamic description of the properties of the radiation, it seems to have gone unnoticed in the past that the conditions encountered by a small object near the horizon may make the calculation of the buoyant force from the fluid point of view inappropriate. Let $R$ denote the typical size of said object; we shall assume $R \ll G M$ ( $M$ is the hole's mass) in order that the full object may be able to approach the horizon. For a Schwarzschild black hole's exterior the exact metric is (units with $c=1$ )

$$
d s^{2}=-\chi^{2} d t^{2}+d z^{2}+r^{2}\left(d \theta^{2}+\sin ^{2} \theta d \phi^{2}\right)
$$

where $z=\int_{2 G M}^{r}(1-2 G M / r)^{-1 / 2} d r$ is the proper radial distance from the horizon while $\chi=(1-2 G M / r)^{1 / 2}$ is the redshift factor mentioned earlier. Our calculations will be done in the region $r-2 G M \ll G M$ (equivalently $z \ll G M$ ); it is only in it that we may approximate Unruh's temperature by Eq.(2), and at the same time ignore Hawking's radiance which is not manifest so near the horizon [2]. We then get

$$
\chi \approx z(4 G M)^{-1} .
$$

In the Schwarzschild case $T_{0}=\hbar(8 \pi G M)^{-1}$, so we have [15, 11

$$
T \approx \hbar(2 \pi z)^{-1}
$$

Thus we expect that the predominant local wavelength in the radiation, $\bar{\lambda}$, at proper distance $z$ from the horizon is roughly of order $z$. In fact, distribution (16) to be obtained below peaks a $\lambda \approx 7.97 z$ and gives the mean wavelength as $\langle\lambda\rangle=14.62 z$. So if the object is near the horizon $(z \ll G M)$ but not nearly touching it $(z \gtrsim R)$, we have $R \lesssim z \sim \bar{\lambda} \ll G M$. At a fundamental level the buoyant force is due to the momentum jolts the object receives as successive waves scatter off it. Waves with $\lambda \gtrsim R$ have difficulty matching specified boundary conditions on the object's surface; hence they tend to scatter poorly and convey little momentum to the object. Waves with $\lambda \ll R$ can match the boundary conditions better, and scatter and convey momentum effectively. However, since these short waves are a minority in the Unruh radiation $(\lambda \ll R \lesssim \bar{\lambda})$, we expect the true buoyant force to be small compared to that we would calculate by means of the radiation pressure (which comes from all wavelengths indiscriminately); in other words, the buoyancy should be nonArchimedean. Detailed calculation in Sec.IIIB confirms this expectation. Since the object being lowered must pass through the region with $z \gtrsim R$ - we call it the far region or FR - the energy accreted by the black hole and its entropy increase when the object is finally dropped in must be influenced by the suppression of buoyancy just mentioned. One purpose of this paper is to correct UW's treatment of the lowering process for the suppression, and to reexamine quantum buoyancy's role in the GSL's operation. 


\section{BUOYANCY IN FAR REGION}

We first concentrate on the situation where the object's bottom is at no time closer (in proper distance) to the horizon than the object's height. The opposite situation will be treated in Sec.[V].

\section{A. Momentum transfer in flat spacetime}

Obviously, a force calculation from wave momentum transfer is bound to be complex as compared to one based on the fluid picture, so we start by discussing the scattering in flat spacetime. It is well known that wave scattering by an object in the long wavelength limit is indifferent to details (target's shape, etc.). The differential crossection has the form

$$
d \sigma / d \Omega=R^{2}(R / \lambda)^{4} F\left(\hat{\mathbf{n}}, \hat{\mathbf{n}}^{\prime}\right)
$$

where $\pi R^{2}$ is a typical geometric crossection of the object, $F$ some dimensionless function, and $\hat{\mathbf{n}}$ and $\hat{\mathbf{n}}^{\prime}$ a pair of unit vectors denoting the incidence and scattering directions, respectively. The fourth order dependence on wavelength comes from the dipole part of the scattering, which predominates at long wavelengths. As an example [19, electromagnetic scattering from a conducting sphere has $F\left(\hat{\mathbf{n}}, \hat{\mathbf{n}}^{\prime}\right)=16 \pi^{2}\left[\frac{5}{8}\left(1+\cos ^{2}\left(\hat{\mathbf{n}} \cdot \hat{\mathbf{n}}^{\prime}\right)\right)-\cos \left(\hat{\mathbf{n}} \cdot \hat{\mathbf{n}}^{\prime}\right)\right]$. If the incident flux of momentum carried in the ambient radiation by wavelengths in the vicinity of $\lambda$ and in the vicinity of the direction $\hat{\mathbf{n}}$ is $\hat{\mathbf{n}} I(\lambda, \hat{\mathbf{n}}) d \lambda d \hat{\mathbf{n}}$, the object will gain momentum at a rate

$$
d \mathbf{P} / d t=\int d \lambda \int d \hat{\mathbf{n}} \hat{\mathbf{n}} I(\lambda, \hat{\mathbf{n}}) R^{2}(R / \lambda)^{4} \int d \hat{\mathbf{n}}^{\prime} F\left(\hat{\mathbf{n}}, \hat{\mathbf{n}}^{\prime}\right)\left(1-\hat{\mathbf{n}} \cdot \hat{\mathbf{n}}^{\prime}\right) .
$$

The factor $1-\hat{\mathbf{n}} \cdot \hat{\mathbf{n}}^{\prime}$ takes into account extra momentum given to the object when a wave backscatters.

Because characteristics of the scattering are insensitive to the object's shape, we may narrow attention to a spherically symmetric object. Results for objects not too far from spherical should be quite similar. In what follows $R$ shall denote the object's radius. The scattering crossection now depends only on the angle of scattering: $F=F\left(\hat{\mathbf{n}} \cdot \hat{\mathbf{n}}^{\prime}\right)$. The integral over the solid angle $\hat{\mathbf{n}}^{\prime}$ is then equivalent to $2 \pi$ times one over $\hat{\mathbf{n}} \cdot \hat{\mathbf{n}}^{\prime}$; it is thus just a number $\xi$, most likely of order unity (for electromagnetic waves $\xi=14 \pi / 3$ ). Thus

$$
d \mathbf{P} / d t=\xi \int d \lambda \int d \hat{\mathbf{n}} \hat{\mathbf{n}} I(\lambda, \hat{\mathbf{n}}) R^{2}(R / \lambda)^{4} .
$$

\section{B. Buoyant force in curved spacetime}

In curved spacetime, particularly in the vicinity of a black hole, we may take over Eq.(8) to an orthonormal frame associated with metric (3) in which the object is at rest. As usual, many quantities, such as field strengths, fluxes, wavelengths, etc. will take on the same values in the orthonormal frame and in a comoving inertial frame; such inertial frame can be large enough to contain the object and its immediate surroundings because by assumption 
the radius of curvature at the horizon, $G M$, is much larger than $R$. This allows us to use flat spacetime results about the scattering. We should reinterpreted $\mathbf{P}, \lambda$ and $t$ above as momentum, wavelength and time measured in the orthonormal frame. And this time must be proper time $\tau$ of the object, while $d \mathbf{P} / d \tau$ stands for the force as measured by a local observer in the orthonormal frame. With this in mind we may rewrite Eq.(8) as

$$
d \mathbf{P} / d \tau=\xi R^{6} \int d \lambda \int d \hat{\mathbf{n}} \hat{\mathbf{n}} I(\lambda, \hat{\mathbf{n}}) \lambda^{-4} .
$$

Since the region including the object and its immediate surroundings is small on scale $G M$, and close to the horizon, $r \approx 2 G M$ there. It proves more convenient to rewrite the metric (3) in said region in the approximate form

$$
d s^{2}=-(z / 4 G M)^{2} d t^{2}+d z^{2}+d x^{2}+d y^{2}
$$

where $d x^{2}+d y^{2}$ is the metric on the almost planar small cap of the sphere $r=$ const. $\approx 2 G M$ which has fixed $z$. The directions $\hat{\mathbf{n}}$ and $\hat{\mathbf{n}}^{\prime}$ have the same components in appropriately oriented inertial and orthonormal frames as with respect to the spacelike coordinate lines of metric $(10)$.

We proceed to calculate $I(\lambda, \hat{\mathbf{n}})$ starting in the global frame defined by Eq.(10). Because that metric is static, the global frequency $\omega_{0}$ of a wave is conserved (being the same over the full black hole exterior described by metric (3)). And because the system is described by a single global temperature, $T_{0}$, one can describe the thermal spectrum by saying that each global mode of a boson field with frequency $\omega_{0}$ is occupied by $\left[\exp \left(\hbar \omega_{0} / T_{0}\right)-1\right]^{-1}$ quanta on average. To find the flux of momentum at a given point we have to enumerate all contributing modes in a small region surrounding the spherical object. To make things as simple as possible consider the scalar equation $\Phi_{, \alpha}{ }^{; \alpha}=0$ on the metric (10):

$$
\Phi_{, u u}+\left(z^{*} / 4 G M\right)^{2} \exp (u / 2 G M)\left(\Phi_{, x x}+\Phi_{, y y}\right)-\Phi_{, t t}=0
$$

Here $u \equiv 4 G M \ln \left(z / z^{*}\right)$, and $z^{*}$ is a reference value of the $z$ coordinate. The symmetries in $x, y$ and $t$ permit a solution of the form

$$
\begin{aligned}
\Phi & =U(u) \exp \left[\imath\left(\kappa_{x} x+\kappa_{y} y-\omega_{0} t\right)\right] \\
0 & =U_{, u u}+\left[\omega_{0}^{2}-\left(z^{*} / 4 G M\right)^{2} \exp (u / 2 G M)\left(\kappa_{x}^{2}+\kappa_{y}^{2}\right)\right] U
\end{aligned}
$$

where $\kappa_{x}, \kappa_{y}$ and $\omega_{0}$ are constants, the last identical to the global frequency mentioned above.

For given $\kappa_{x}$ and $\kappa_{y}$ there are oscillatory solutions of Eq.(13) for sufficiently large $\omega_{0}$. In WKB approximation they take the form

$$
\begin{aligned}
U & \approx \exp \left\{\imath \int\left[\omega_{0}^{2}-\left(z^{*} / 4 G M\right)^{2} \exp (u / 2 G M)\left(\kappa_{x}^{2}+\kappa_{y}^{2}\right)\right]^{1 / 2} d u\right\} \\
& =\exp \left\{\imath \int\left[\omega_{0}^{2}(4 G M / z)^{2}-\left(\kappa_{x}^{2}+\kappa_{y}^{2}\right)\right]^{1 / 2} d z\right\}
\end{aligned}
$$

From the last form, which is $z^{*}$ independent, we infer the effective wavevector component in the $z$ direction: $\kappa_{z}=\left[\omega_{0}^{2}(4 G M / z)^{2}-\left(\kappa_{x}^{2}+\kappa_{y}^{2}\right)\right]^{1 / 2}$. Although the accelerated character of the global coordinates causes this component to vary, over the short $z$ interval encompassed 
by the sphere we can think of $\left\{\kappa_{x}, \kappa_{y}, \kappa_{z}\right\}$ as a wavevector. Obviously the corresponding wavelength is $\lambda=2 \pi\left(\kappa_{x}^{2}+\kappa_{y}^{2}+\kappa_{z}^{2}\right)^{-1 / 2}=\pi z\left(2 G M \omega_{0}\right)^{-1}$, which is just what we would have gotten had we corrected the global frequency $\omega_{0}$ for the redshift $\chi$. $\lambda$ thus coincides with the locally measured wavelength we have been using intuitively.

Now according to Eq.(10), $x, y$ and $z$ measure proper length; therefore, per unit proper volume, the number of allowed values of $\kappa_{x}, \kappa_{y}$ and $\kappa_{z}$ in an interval $d \kappa_{x}, d \kappa_{y}$ and $d \kappa_{z}$ is given by $(2 \pi)^{-3} d \kappa_{x} d \kappa_{y} d \kappa_{z}$, or equivalently, by $\lambda^{-4} d \hat{\mathbf{n}} d \lambda$, where $d \hat{\mathbf{n}}$ is the solid angle spanned by the various wavevectors. Taking two helicities for each wavevector (here we pass from scalar waves to electromagnetic), and remembering that the momentum carried by a singly occupied mode, as measured in the orthonormal frame, is $2 \pi \hbar \lambda^{-1}$, we obtain the momentum flux density $I(\lambda, \hat{\mathbf{n}})$ in the orthonormal frame,

$$
I(\lambda, \hat{\mathbf{n}})=\frac{4 \pi \hbar \lambda^{-5}}{e^{\frac{2 \pi \hbar}{\lambda T}}-1}
$$

Here we have used Eq.(2) to replace $\hbar \omega_{0} / T_{0}$ by $2 \pi \hbar(\lambda T)^{-1}$.

We should not be fooled by the similarity of this expression to the Planckian distribution in textbooks. Because of the variation of the redshift $\chi, \lambda$ of a particular mode changes (redshifts) as the wave propagates (and thus passes from one orthonormal frame to a neighboring one). Alternatively, if we ask how much momentum is carried by the radiation in $(\lambda, \lambda+d \lambda)$, we must be aware that $T$ in Eq.(16) varies with position according to Eq.(价). This has implications for the integral over $\hat{\mathbf{n}}$ in Eq.(9). The temperature $T$ to be used therein is not isotropic because it is not meant to be taken at a fixed point. A (plane) wavefront incident on the sphere with direction $\hat{\mathbf{n}}$ first meets its surface at coordinate $z=z_{c}-\hat{\mathbf{z}} \cdot \hat{\mathbf{n}} R$, ( $\hat{\mathbf{z}}$ is a unit vector in the positive $z$ direction and $z_{c}$ is the sphere center's $z$ coordinate). Therefore, it is reasonable to evaluate the temperature governing the incident radiance's intensity at this $z$. In view of Eq.(2), we must take

$$
T=(\hbar / 2 \pi)\left(z_{c}-\hat{\mathbf{z}} \cdot \hat{\mathbf{n}} R\right)^{-1}
$$

in Eq.(16) when evaluating the integral in Eq.(9).

We do the $\lambda$ integral first. Of course, the dependence $\lambda^{-4}$ assumed for the crossection is not valid for $\lambda \lesssim R$. But according to Eq.(2), the exponential appearing in Eq.(16), $\exp \left(4 \pi^{2} z / \lambda\right)$, is large when $\lambda<R$ because by assumption $R<z$ : the Planckian distribution suppresses contributions to $d \mathbf{P} / d \tau$ from short wavelengths. Thus we may extend the integration range down to $\lambda=0$ while incurring only a small error. After rescaling and using the identity $\int_{0}^{\infty} x^{7}\left(e^{x}-1\right)^{-1} d x=8 \pi^{8} / 15$ we get

$$
\frac{d \mathbf{P}}{d \tau}=\frac{\xi \hbar R^{6}}{30720 \pi^{7}} \int \frac{\hat{\mathbf{n}} d \hat{\mathbf{n}}}{\left(z_{c}-\hat{\mathbf{z}} \cdot \hat{\mathbf{n}} R\right)^{8}}
$$

By symmetry the integral must be proportional to $\hat{\mathbf{z}}$; it is easily evaluated by going over to the variable $\hat{\mathbf{z}} \cdot \hat{\mathbf{n}}$. To get the total FR buoyant force as measured at infinity, $\mathbf{f}_{>}$, one must multiply $d \mathbf{P} / d \tau$, the buoyant force measured locally, by $d \tau / d t$ of the sphere, which equals $\chi \approx z_{c}(4 G M)^{-1}$. We also multiply by $N$, the effective number of species of quanta in the radiation, assuming the object scatters all (photons contribute unity; because radiations of different kinds scatter differently, $N$ is not necessarily an exact integer). Thus, 


$$
\mathbf{f}_{>}=\frac{\hat{\mathbf{z}} N \xi \hbar R^{7}}{11520 \pi^{6} G M} \frac{z_{c}^{6}+2 R^{2} z_{c}^{4}+3 R^{4} z_{c}^{2} / 7}{\left(z_{c}^{2}-R^{2}\right)^{7}} .
$$

Although we have carried out all integrals exactly, it must be understood that Eq.(19) may already become inaccurate for $z_{c} \lesssim 2 R$ because in that region the condition $\lambda \gg R$ is satisfied only marginally (as mentioned we expect $\bar{\lambda} \sim 10 z_{c}$ ). The region $z_{c} \lesssim 2 R$ will be examined in Sec.IV.

We have just found that for $z_{c} \gg R, \mathbf{f}_{>} \propto z_{c}^{-8}$. By contrast, the force calculated a la UW's fluid approach, Eq.(31) below, would behave in that region as $z_{c}^{-4}$. The corrections to the fluid approach's results are thus, a priori, nontrivial in the FR.

\section{Energetics in far region and GSL}

Following UW we calculate the energy of the object as it is slowly lowered from infinity. As in many previous papers [2, 1, 12 [15], we assume that the object's proper energy $E$ and its entropy $S$ are unaffected by the descent. The object's gravitational energy at any stage is $E_{\text {grav }}=E \chi \approx E z_{c}(4 G M)^{-1}$. The contribution to the energy from the work done to overcome the buoyancy is $\int_{z_{c}}^{\infty} \hat{\mathbf{z}} \cdot \mathbf{f}_{>} d z_{c}$. Of course our expression (19) is not reliable for $z_{c} \gtrsim G M$; however, the buoyant force drops off so fast with $z_{c}$ that we make a small error if w use Eq.(19) all the way to infinity. Doing the integral exactly we find the total energy function

$$
\mathcal{E}_{>}\left(z_{c}\right)=\frac{E z_{c}}{4 G M}+\frac{\xi N \hbar R^{7} z_{c}^{3}\left(z_{c}^{2}+R^{2}\right)}{80640 \pi^{6} G M\left(z_{c}^{2}-R^{2}\right)^{6}} .
$$

As UW point out, the most stringent test of the GSL is had by dropping the object from the floating point where $\mathcal{E}_{>}$reaches its minimum. This point is situated at $z_{c}=\ell R$ with $\ell$ the positive root of

$$
\begin{gathered}
\frac{\left(\ell^{2}-1\right)^{7}}{\ell^{6}+2 \ell^{4}+3 \ell^{2} / 7}=\frac{\xi \sigma^{2}}{16 \pi^{5}} \\
\sigma \equiv(N \hbar / 180 \pi E R)^{1 / 2}
\end{gathered}
$$

We shall find $\sigma$ to be the main parameter delimiting various buoyancy regimes. The following flat spacetime intuition of it is useful. Consider an object of radius $R$ and energy $E$ immersed in thermal radiation comprising $N$ species whose energy density equals the object's energy density $\sim E / R^{3}$. The usual Boltzmann formulae allow us to conclude that the typical wavelength $\bar{\lambda}$ is of order $\sqrt{ } \sigma R$. Thus a fluid (continuum) description of the radiation's interaction with the object will be a good one for $\sigma \ll 1$. If the object is macroscopic or even mesoscopic, its Compton length $\hbar / E$ must be much smaller than $R$, so the requirement on $\sigma$ will hold nicely provided $N$ is not large.

In the black hole spacetime, for the floating point to be in the FR means $\ell \gtrsim 2$. According to Eq.(21) this requires $\sigma \gtrsim 10^{2}$, definitely not the fluid regime as we have realized already. This large a $\sigma$ requires $N$ of the Unruh radiance to be very large ( $10^{6}$ at least). Although we are only aware of relatively few particle species in nature, it is of interest, as stressed by UW, to check whether the GSL would continue to work in the face of a proliferation of radiance species. 
UW propose (step 2 in Sec.I here) that the floating point occurs precisely where $E$ equals the proper energy of the displaced radiation $\int e d x d y d z$. The energy density of radiation corresponding to spectrum (16) has the textbook form $e=\left(N \pi^{2} T^{4} / 15 \hbar^{3}\right)$. We substitute Eq.(2) and integrate over a spherical volume equal to the sphere's and at the same height above the horizon. Since the horizontal crossection of the sphere at height $z$ is $\pi\left[R^{2}-\left(z_{c}-z\right)^{2}\right]$, the integral becomes

$$
\frac{N \hbar}{240 \pi^{2}} \int_{z_{c}-R}^{z_{c}+R} \frac{\pi\left[R^{2}-\left(z-z_{c}\right)^{2}\right]}{z^{4}} d z=\frac{N \hbar R^{3}}{180 \pi\left(z_{c}^{2}-R^{2}\right)^{2}}
$$

This equals $E$ at $z_{c}=R \sqrt{1+\sigma}$ which is distinct from $R \ell$ : contrary to UW's step 2, here the equal-energies point is distinct from the floating point, and Archimedes' principle is invalid. Thus the buoyancy in the FR may be termed non-Archimedean.

This setback does not by itself disable the UW program. The overall argument might still work if one could prove that the minimum $\Delta S_{B H}$ exceeds the entropy contained in a spherical volume of Unruh radiance equal to the object's and centered at $z_{c}=R \sqrt{1+\sigma}$ where it has the same proper energy as the object. We call this last the displaced entropy, $\mathcal{S}_{\text {disp }}$, and evaluate it by integrating $s=(4 e / 3 T)$ over said spherical volume. In terms of $h(x) \equiv \ln (\sqrt{1+x}+1)-\ln (\sqrt{1+x}-1)$,

$$
\mathcal{S}_{\text {disp }}=(N / 90)\left[2 \sigma^{-1} \sqrt{1+\sigma}-h(\sigma)\right]
$$

Now in terms of $\ell$ and $\sigma^{2}, \mathcal{E}_{>}(\ell R)$ is easily rewritten as

$$
\mathcal{E}_{>}(\ell R)=\frac{E R}{4 G M}\left[\ell+\frac{\xi \sigma^{2}}{112 \pi^{5}} \frac{\ell^{3}\left(\ell^{2}+1\right)}{\left(\ell^{2}-1\right)^{6}}\right]
$$

Expressing $E R$ by means of Eq.(22) and $\xi \sigma^{2}$ inside the square brackets by means of Eq.(21), and dividing by $T_{0}$ gives the minimum increase in black hole entropy:

$$
\Delta S_{B H}=\frac{N}{90 \sigma^{2}} \frac{\ell\left(8 \ell^{4}+14 \ell^{2}+2\right)}{7 \ell^{4}+14 \ell^{2}+3}
$$

A plot of both $\Delta S_{B H}$ and $\mathcal{S}_{\text {disp }}$ as functions of $\ell$ with $\sigma$ determined by Eq.(21) shows that actually $S_{B H}<\mathcal{S}_{\text {disp }}$ already for $\ell>1.24$ (we took $\xi=10$ but the results change little over a large range of $\xi$ ). Thus the inequality required by UW is violated over the whole FR where buoyancy has to be treated as due to wave scattering.

This being the case, the only way one could establish the GSL's validity from the effect of the buoyancy would be to show that $\mathcal{S}_{\text {disp }}$ is not just as large as $S$, but sufficiently larger so as to compensate for the failure of $\Delta S_{B H}>\mathcal{S}_{\text {disp }}$ whenever $\sigma$ is not small. This can no longer follow from a general principle such as UW's entropy bound, but would entail looking into details of the object, something quite opposite to UW's intent to establish the GSL in general. We conclude that in the FR UW's argument that buoyancy automatically enforces the GSL for any $N$ cannot be completed.

On the other hand, if we substitute $\sigma^{2}$ from Eq.(22) into Eq.(26), and observe that the function of $\ell$ appearing in this last one exceeds unity for $\ell>1$, we conclude that

$$
\triangle S_{B H}>2 \pi E R / \hbar
$$

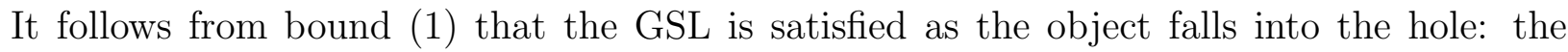
decrease of entropy by $S$ is compensated by the gain $\Delta S_{B H}$. Bound (11) is thus a sufficient condition for the operation of the GSL when the floating point is in the FR. 


\section{BUOYANCY IN NEAR-HORIZON REGION}

We now look at the situation where the object's bottom eventually comes much closer to the horizon than the object's radius, so that it is in the near-horizon region (NHR). Because $\bar{\lambda}$ for waves hitting the bottom is of order $10\left(z_{c}-R\right)$, and we shall want to keep it small compared to $R$, it is convenient to define the NHR as $R<z_{c} \lesssim 1.01 R$.

\section{A. The buoyant force}

With the sphere so positioned the wavelengths of waves hitting a cap at the sphere's bottom $\left(\lambda \sim z_{c}-R\right)$ are short compared to the cap's transversal size $\sim R$. In this case the fluid model can be used to compute the force on the cap. As we go up the sphere's surface, the waves hitting it get longer and eventually are no longer short compared to the scale of the surface. Somewhere along the side of the sphere the fluid model fails. Simultaneously, as we go up the surface, the radiation (fluid) pressure

$$
p=\frac{N \pi^{2} T^{4}}{45 \hbar^{3}}=\frac{N \hbar}{720 \pi^{2} z^{4}}
$$

drops precipitously as $z$ grows. Thus if we formally integrate the pressure force all around the sphere, the main contribution comes from the cap of about a steradian in size at the bottom which is almost horizontal and very close to the horizon everywhere. And this is precisely the part of the force which is well described by the fluid model. The force should be corrected for the contribution from the rest of the sphere which is in the wave scattering regime. However, we know that the wave scattering force tends to be weaker than the fluid force. Hence, our integral must give a close approximation to the true force. The preceding comments amount to a justification, when $z_{c}-R \ll R$, of UW's method for calculating the buoyant force.

As UW mention, the pressure has to be multiplied by $\chi$ before integration, so that the force will be "as measured at infinity". Therefore, working in metric (10), the force in the NHR is

$$
\mathbf{f}_{<}=-\oint \chi p d \mathbf{S}=-\int \nabla(\chi p) d x d y d z
$$

We have used Gauss' theorem to convert the integral into one over the volume the radiation would have occupied but for the sphere's presence. As in Eq.(23) we rewrite this as

$$
-\int_{z_{c}-R}^{z_{c}+R} \nabla(\chi p) \pi\left[R^{2}-\left(z-z_{c}\right)^{2}\right] d z=-2 \pi \hat{\mathbf{z}} \int_{z_{c}-R}^{z_{c}+R} \chi p\left(z-z_{c}\right) d z
$$

where we have done an integration by parts. Substituting Eqs.(28) and (4) gives

$$
\mathbf{f}_{<}=\frac{\hat{\mathbf{z}} N \hbar}{720 \pi G M} \frac{R^{3}}{\left(z_{c}^{2}-R^{2}\right)^{2}}
$$




\section{B. Energetics in near-horizon region and GSL}

The contribution to the energy of the sphere from the work done against the buoyancy is formally $\int_{z_{c}}^{\infty} \hat{\mathbf{z}} \cdot \mathbf{f} d z_{c}$, where $\mathbf{f}$ denotes the exact buoyant force. If we add and substract $\mathbf{f}_{<}$ to $\mathbf{f}$, perform the integral $\int_{z_{c}}^{\infty} \hat{\mathbf{z}} \cdot \mathbf{f}_{<} d z_{c}$, and then take into account the gravitational energy as in Eq.(20), we get

$$
\begin{aligned}
\mathcal{E}_{<}\left(z_{c}\right) & =\frac{E z_{c}}{4 G M}+\frac{N \hbar}{2880 \pi G M}\left[\frac{2 R z_{c}}{z_{c}^{2}-R^{2}}-\ln \left(\frac{z_{c}+R}{z_{c}-R}\right)\right]-\mathcal{C} \\
\mathcal{C} & \equiv \int_{z_{c}}^{\infty} \hat{\mathbf{z}} \cdot\left(\mathbf{f}_{<}-\mathbf{f}\right) d z_{c}
\end{aligned}
$$

Because $\mathbf{f}_{<} \rightarrow \mathbf{f}$ in the NHR, $\mathcal{C}$ is actually constant so long as $z_{c}$ is taken in the NHR.

Because outside the NHR part of the the buoyancy is best described by wave scattering, and should be weaker than when calculated entirely from the fluid model, we may infer that $\mathcal{C}>0$. A fair approximation to $\mathcal{C}$ can be had by replacing $\mathbf{f}$ in Eq.(33) by $\mathbf{f}_{>}$over the range of $z_{c}$ for which $\mathbf{f}_{<} \geq \mathbf{f}_{>}$, and setting $\mathbf{f}=\mathbf{f}_{<}$in the domain where $\mathbf{f}_{<}<\mathbf{f}_{>}$. By comparing Eqs.(19) and (31) numerically one finds that the transition occurs at $z_{c}=1.19826 R$ for $\xi=10$, with almost no change as $\xi$ varies by factors of 10 either way. Using these values a numerical integration gives $\mathcal{C}=0.00661(N \hbar / 8 \pi G M)$, confirming our hunch about the sign of $\mathcal{C}$, but also showing that in natural units $\mathcal{C}$ is small. Hence, the fact that $\mathcal{C}$ has only been approximated should not upset the following arguments.

The floating point, determined by the minimum of $\mathcal{E}_{<}\left(z_{c}\right)$, is unaffected by the value of $\mathcal{C}$; it now occurs at $z_{c}=\ell R$ with

$$
\ell=\sqrt{1+\sigma}
$$

and $\sigma$ still defined by Eq.(22). For self-consistency this should fall within the NHR: $\ell<1.01$. By Eq.(34) we thus require $\sigma<0.02$. This condition is fulfilled by all macroscopic or mesoscopic objects $(\hbar / E \ll R)$ unless the number of radiance species $N$ is very large. Position (34) coincides with that at which the object displaces its own energy's worth of radiation, as we saw in Sec.IIC. Thus in the NHR the buoyancy is Archimedean, and step 2 of UW's argument is upheld.

The growth in black hole entropy when the sphere is dropped from the floating point, namely $T_{0}{ }^{-1} \mathcal{E}_{<}(\sqrt{1+\sigma} R)$, is

$$
\Delta S_{B H}=(N / 90)\left[\sigma^{-2}(1+\sigma / 2) \sqrt{1+\sigma}-h(\sigma) / 4-0.595\right]
$$

The displaced entropy is given by Eq.(24). It is easy to see that for $\sigma<0.02, \Delta S_{B H}>\mathcal{S}_{\text {disp. }}$. We thus confirm step 1 of UW's argument.

Step 3 in UW's procedure maintains that $\mathcal{S}_{\text {disp }}>S$ on the grounds that unconfined thermal radiation is the most entropic form of matter for given volume and energy. This generic entropy bound has been questioned [14] because the two entities compared here, unconfined thermal radiation, and matter confined to the object, are subject to different boundary conditions. Although violations of $\mathcal{S}_{\text {disp }}>S$ are rampant for systems whose various dimensions are very different, they have not been found for spherical ones. Accepting the inequality in the spirit of this paper's scope, we find with UW that if the floating point 
lies in the NHR, $\Delta S_{B H}>S$. This, of course, guarantees that the GSL will work as the spherical object falls in.

In the above argument it is the quantum buoyancy which protects the GSL. However, it does not follow, as UW [2] would have it, that one cannot derive the entropy bound (1) from the GSL, nor as PW [12 would have it, that the entropy bound holds only because of additional assumptions about the magnitude of $N$ and $\hbar(R E)^{-1}$. In this section we only assume that $\sigma \ll 1$, and this in order that $\ell-1 \ll 1$, so that the fluid method is accurate.

Let us now replace the $N$ in Eq.(35) in terms of $\sigma^{2}$ according to Eq.(22). We find

$$
\Delta S_{B H}=(2 \pi R E / \hbar)\left[(1+\sigma / 2) \sqrt{1+\sigma}-(1 / 4) h(\sigma) \sigma^{2}-0.595 \sigma^{2}\right]
$$

Since the last two terms in the square brackets are negative, the GSL will fail upon infall of the object unless at least

$$
S<(2 \pi R E / \hbar)(1+\sigma / 2) \sqrt{1+\sigma}
$$

For the small $\sigma$ we are assuming this is equivalent to the entropy bound (1), which is thereby seen to be a necessary condition for the GSL. We further discuss this derivation of the bound in Sec. (V. Since it is carried out in the face of buoyancy, it serves as justification for several recent extensions of the bound to spinning [20] and charged [21] entropy-bearing objects whose derivations ignored buoyancy.

We also note that the function of $\sigma$ in the square brackets in Eq.(36) is positive for $\sigma<0.02$. Thus the entropy bound (1) guarantees that $\Delta S_{B H}>S$ : when the floating point is in the NHR, the entropy bound is also a sufficient condition for operation of the GSL.

\section{THE INTERMEDIATE REGION}

In the intermediate region $1.01 R \lesssim z_{c} \lesssim 2 R$ neither of the previous calculations of the buoyant force is expected to be accurate. To calculate the buoyant force directly from scattering without benefit of the approximationS $\lambda \gg R$ or $\lambda \ll R$ would be very difficult. Thus we propose to substitute $\mathbf{f}$ in the intermediate region by an interpolation between $\mathbf{f}_{>}$ and $\mathbf{f}_{<\cdot}$. Specifically, we interpolate harmonically between the two versions of the buoyancy energy, to wit

$$
\mathcal{E}\left(z_{c}\right)=\frac{E z_{c}}{4 G M}+\frac{\tilde{\mathcal{E}}_{<}\left(z_{c}\right) \tilde{\mathcal{E}}_{>}\left(z_{c}\right)}{\tilde{\mathcal{E}}_{<}\left(z_{c}\right)+\tilde{\mathcal{E}}_{>}\left(z_{c}\right)} ; \quad \tilde{\mathcal{E}}_{j} \equiv \mathcal{E}_{j}-\frac{E z_{c}}{4 G M}
$$

The $\mathcal{E}_{j}$ 's here are as defined by Eqs.(20) and (32), the last with $\mathcal{C}$ set to zero since we found it to be relatively small. This interpolation for $\mathcal{E}$ is completely smooth, and obviously has the correct asymptotics for $z_{c}-R \ll R$ and $z_{c} \gg R$. Numerically we find that $-\partial \mathcal{E} / \partial z_{c}$ agrees with $\mathbf{f}_{<}$to at least $0.001 \%$ for $R<z_{c}<1.01 R$, and with $\mathbf{f}_{>}$to at least $0.05 \%$ for $z_{c}>2 R$. Eq.(38) should thus be a reasonable good approximation to $\mathcal{E}$ in the intermediate region.

The interpolated $\mathcal{E}$ can be cast into the form 


$$
\begin{aligned}
\mathcal{E}\left(z_{c}\right) & =E R(4 G M)^{-1} \mathcal{F}\left(\xi, \sigma^{2}, z_{c} / R\right) \\
\mathcal{F}\left(\xi, \sigma^{2}, v\right) & \equiv v+\frac{\xi \sigma^{2}\left(v^{5}+v^{3}\right) g(v)}{4\left(v^{2}-1\right)\left[\xi\left(v^{5}+v^{3}\right)+28 \pi^{5}\left(v^{2}-1\right)^{5} g(v)\right]} \\
g(v) & \equiv 2 v-\left(v^{2}-1\right) \ln \left(\frac{v+1}{v-1}\right)
\end{aligned}
$$

$\mathcal{F}$ as a function of $v=z_{c} / R$ has a single minimum in the physical range $v>1$. Thus $\mathcal{E}$ is minimal at a value of $z_{c} / R$ - we again denote it $\ell$ - which depends only on $\sigma$ and $\xi$. Taking $\xi=10$ we find numerically that $\ell$ increases monotonically with $\sigma$. In particular, it grows from 1.01 to 2 as $\sigma$ varies from 0.02 to 105 . In light of previous findings, the corresponding range of $z_{c}$ bridges the gap between NHR and FR.

Replacing $E R$ in Eq.(39) by means of Eq.(22) and dividing by $T_{0}$ gives the minimum increase in black hole entropy:

$$
\Delta S_{B H}=\frac{N}{90 \sigma^{2}} \mathcal{F}\left(\xi, \sigma^{2}, \ell(\xi, \sigma)\right)
$$

This has to be compared with $S_{\text {disp }}$ of Eq.(24). Numerically we find (again for $\xi=10$ ) that $\Delta S_{B H} \leq S_{\text {disp }}$ for $\sigma \geq 1.42$, which contradicts step 1 of UW's argument. The transition point $\Delta S_{B H}=S_{\text {disp }}$ occurs here at $\ell=1.275$, a position close to the $\ell=1.24$ that we computed for the switch in Sec.III using FR formulae. As already discussed there, under the circumstances one cannot naturally redesign the buoyancy argument to understand why the GSL works. That argument is of no help when $N$ is large enough to make $\sigma$ somewhat larger than unity.

How does the entropy bound (11) fare? Evaluating $\sigma$ in the prefactor of Eq.(12) we get

$$
\Delta S_{B H}=(2 \pi E R / \hbar) \mathcal{F}\left(\xi, \sigma^{2}, \ell(\xi, \sigma)\right)
$$

Numerically $\mathcal{F}\left(\xi, \sigma^{2}, \ell(\xi, \sigma)\right)>1$ for $\ell>1$. Hence, also in the intermediate $\sigma$ regime $(1.01 \lesssim \ell \lesssim 2)$, bound (11) implies the inequality $\Delta S_{B H}>S$, and is thus a sufficient condition for the operation of the GSL when the object is dropped in.

To what extent is the entropy bound also a necessary condition for the GSL: can the bound be derived from the GSL ? Numerically we find $\mathcal{F}\left(\xi, \sigma^{2}, \ell(\xi, \sigma)\right)<1.1$ for $\sigma<0.1$. Applying the GSL to our gedanken experiment allows us to derive bound (11) for $\sigma<0.1$, apart from a $<10 \%$ correction. We may reach the same conclusion from Eq.(37). Bound (1) can also be derived for arbitrary $N$, apart from an overall constant of crudely order unity, by considering free fall of the object into a black hole [16]. Since in all cases where $S$ can be computed explicitly for non-black hole systems [5], one finds the true $S$ to always fall at least an order of magnitude below bound (11), there is no point in introducing corrections to

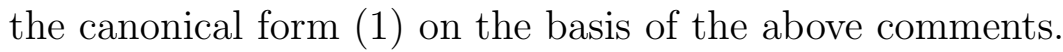

\section{BUOYANCY OF A CHARGED ELEMENTARY PARTICLE}

All our previous considerations referred to macroscopic spherical objects. For $N$ not large these have very small $\sigma$, so that only the NHR case is important (if we exclude the issue of calculating the offset $\mathcal{C}$ ). It is, however, interesting to enquire into buoyancy of an 
elementary particle, one whose radius is of order of its Compton length, $\hbar / E$. The particle can no longer be regarded as a sphere with reflecting walls; wave scattering from it must be described by a coupling of the waves with the particle's interaction Hamiltonian. This is obviously detail dependent, and complicated in general. But in the special case of a structureless electrically charged particle, the (Compton) scattering is well understood.

The characteristic length associated with a singly charged particle is the Thomson radius $R_{\mathrm{T}}=e^{2} / E$. Because $\alpha \equiv e^{2} / \hbar \approx 1 / 137, R_{\mathrm{T}} \ll \hbar / E$, so if it were possible to lower the particle down to the horizon, its center's position $z_{c} \approx \hbar / E$ would still be a few lengths $R_{\mathrm{T}}$ away from it. In this sense the particle is always in the FR. But, as we shall see, the buoyant force is of NHR form. The point is that for wavelengths $\lambda \gg \hbar / E$, and averaged over the two polarizations, the scattering crossection is

$$
d \sigma / d \Omega=\left(R_{\mathrm{T}}^{2} / 2\right)\left[1+\left(\hat{\mathbf{n}} \cdot \hat{\mathbf{n}}^{\prime}\right)^{2}\right]
$$

where $\hat{\mathbf{n}}$ and $\hat{\mathbf{n}}^{\prime}$ are the incidence and scattering directions, respectively. This form is missing the $(R / \lambda)^{4}$ of Eq.(6); this explains why FR behavior does not set in.

Instead of Eq.(可) we have

$$
\begin{aligned}
d \mathbf{P} / d t & =\left(R_{\mathrm{T}}{ }^{2} / 2\right) \int d \lambda \int d \hat{\mathbf{n}} \hat{\mathbf{n}} I(\lambda, \hat{\mathbf{n}}) \int d \hat{\mathbf{n}}^{\prime}\left[1+\left(\hat{\mathbf{n}} \cdot \hat{\mathbf{n}}^{\prime}\right)^{2}\right]\left(1-\hat{\mathbf{n}} \cdot \hat{\mathbf{n}}^{\prime}\right) \\
& =\left(8 \pi R_{\mathrm{T}}^{2} / 3\right) \int d \lambda \int d \hat{\mathbf{n}} \hat{\mathbf{n}} I(\lambda, \hat{\mathbf{n}}) .
\end{aligned}
$$

Substituting Eq.(16) for $I$, doing first the $\lambda$ integral by rescaling and use of the identity $\int_{0}^{\infty} x^{3}\left(e^{x}-1\right)^{-1} d x=\pi^{4} / 15$, and identifying $t$ with the proper time $\tau$ in the orthonormal frame leads us to $(N=1$ here by assumption: the particle interacts only with electromagnetic waves)

$$
d \mathbf{P} / d \tau=\left(32 \pi^{6} \hbar R_{\mathrm{T}}^{2} / 45\right) \int d \lambda \int d \hat{\mathbf{n}} \hat{\mathbf{n}}(T / 2 \pi \hbar)^{4} .
$$

As explained at the end of Sec.[IIB, one must take $T$ as anisotropic, depending on the direction of incidence; since $R_{\mathrm{T}}$ is the characteristic radius of scattering here, it replaces $R$ in the expression for $T$, Eq.(17), which we substitute in Eq.(47). The integral over $\hat{\mathbf{n}}$ is performed as in Sec.IIIB. Multiplying the result by $d \tau / d t=\chi \approx z_{c}(4 G M)^{-1}$ we get the buoyant force as measured at infinity

$$
\mathbf{f}=\frac{\hat{\mathbf{z}} \hbar}{270 \pi G M} \frac{R_{\mathrm{T}}^{3} z_{c}^{2}}{\left(z_{c}^{2}-R_{\mathrm{T}}^{2}\right)^{3}} .
$$

Because $z_{c} \gtrsim \hbar / E \gg R_{\mathrm{T}}$, this force is of the same form as Eq.(31) for a macroscopic sphere in the NHR, if we replace $R \rightarrow R_{\mathrm{T}}$.

Several caveats about this derivation are in order. We have neglected quantum corrections to the Thomson crossection (44) which are given by the Klein-Nishina formula. These corrections become important when $\lambda \lesssim 2 \pi \hbar / E$. As we saw in Sec.⿴囗十, $\bar{\lambda} \sim 10 z$, and so as the particle approaches the horizon $\left(z_{c} \sim \hbar / E\right)$, neglect of these corrections is no longer entirely justified. However, further out Eq.(48) should be accurate in this respect. We have also neglected quantum corrections arising from the fact that by the uncertainty principle, the 
particle cannot be at rest and well localized at once, as we assume implicitly when we appeal to buoyancy. Further, the force (48) can only represent an average: the particle should be subject to buffeting originating in thermal fluctuations of the Unruh radiance.

Because $z_{c} \gg R_{\mathrm{T}}=\alpha(\hbar / E)$, we can recast Eq.(48) into the form

$$
\mathbf{f} \approx(2 / 135 \pi) \alpha^{3}(\hbar / E z)^{4} \mathbf{f}_{\text {grav }},
$$

where $\mathbf{f}_{\text {grav }}=\hat{\mathbf{z}} E(4 G M)^{-1}$ is the gravitational force as measured at infinity. Obviously, $|\mathbf{f}| \ll\left|\mathbf{f}_{\text {grav }}\right|$ over the physical range $z_{c}>\hbar / E$. Hence buoyancy is negligible for our particle; there is no floating point, and buoyancy corrections to the energetics are entirely negligible. The neglected quantum corrections are unlikely to change this extreme state of affairs. In recent arguments supporting the uniformly spaced black hole area quantum spectrum [16 18, the buoyancy of an elementary particle was ignored. The present work supplies a basis for that neglect.

\section{DISCUSSION}

The effects of quantum buoyancy are parametrized by $\sigma$ defined in Eq.(22). In a world with few radiation species, $\sigma \approx 10^{-2}, 10^{-5}$, and $10^{-18}$ for an average nucleus, an average atom, and a grain of salt, respectively. All these objects would thus have floating points almost at the horizon $\left(z_{c}-R \ll R\right)$. The results of Secs. [VB and $\nabla$ then tell us that buoyancy protects the GSL upon infall of these or larger objects into a black hole. They also allow us to derive the universal entropy bound (1) from the GSL, apart from a tiny correction which can probably be neglected. For macroscopic and mesoscopic objects, the entropy bound is thus a necessary condition for the operation of the GSL, provided the number of radiant species $N$ is not very large.

As the number of radiation species $N$ is increased, $\sigma$ scales as $\sqrt{ } N$, and will eventually get to be a few tenths of unity for any specified object. The entropy bound for that object cannot then be derived from the GSL as done previously. It stops being a necessary condition for the GSL, but remains a sufficient condition for the law to function, this for arbitrary $N$ or $\sigma$. Although it seems highly unlikely that the world's particle spectrum contains very many species, UW and PW have urged to consider what keeps the GSL working in such a hypothetical eventuality. Their suggestion that buoyancy does the job for any $N$ is overturned by our finding (Sec. $\nabla)$ that for $\sigma$ exceeding $1.42\left(N>10^{9}\right.$ for an atomic sized system), buoyancy becomes insufficient to protect the GSL.

The fact that the universal entropy bound remains a sufficient condition for the GSL when $N$ gets large motivates us to consider the bound as the more fundamental principle. This is in the spirit of today's outlook that holographic-type principles are very deep. For weakly gravitating systems the entropy bound implies the standard holographic principle [8.9]. Neither entropy bound nor holographic principle refers to the number of particle species in nature. One might worry that this is wrong: if one has more species to split a fixed energy among, should not the possible entropy be higher the higher $N$ ? But if this were true, the arguments at the end of Sec.III and at the end of Sec. $\square$ showing that the entropy bound guarantees that the generalized entropy grows upon accretion of the object by a black hole could fail. 
We thus retain the standard form (11) of the bound. In our opinion, the absence of $N$ from the bound, from the holographic principle, and from the formula for black hole entropy, can have two possible origins. One is that the deep logic of physical theory requires a specific particle spectrum and a definite - not too large - $N$ for which the black hole entropy attains its accepted value, and for which entropy bound and holographic principle are still respected, growth of entropy with $N$ notwithstanding. The second possibility is that a proliferation of particle species would entail interactions between them to such an extent that it would change the way in which entropy scales up with $N$ for noninteracting species, causing it to saturate at large $N$ at a value consistent with entropy bound and holographic principle.

\section{ACKNOWLEDGMENTS}

This research is supported by a grant from the Israel Science Foundation, established by the Israel Academy of Sciences and Humanities.

[1] W. G. Unruh, Phys. Rev. D14, 870 (1976).

[2] W. G. Unruh and R. M. Wald, Phys. Rev. D25, 942 (1982); D27, 2271 (1983).

[3] J. D. Bekenstein, Phys. Rev. D7, 2333 (1973); 9, 3292 (1974).

[4] J. D. Bekenstein, Phys. Rev. D23, 287 (1981).

[5] J. D. Bekenstein and M. Schiffer, Int. J. Mod. Phys. C 1, 355 (1990).

[6] G. 't Hooft, in Salam-festschrifft, ed. A. Aly, J. Ellis and S. Randjbar-Daemi (World Scientific, Singapore 1993), gr-qc/9310026.

[7] L. Susskind, J. Math. Phys. 36, 6377 (1995).

[8] J. D. Bekenstein, in Proceedings of the VII Marcel Grossmann Meeting on General Relativity, eds. R. T. Jantzen and G. M. Keiser (World Scientific, Singapore, 1996), p.39, gr-qc/9409015.

[9] R. Bousso, hep-th/9905177 and hep-th/9906022.

[10] L. X. Li and L. Liu, Phys. Rev. D46, 3296 (1992).

[11] O. Zaslavskii, Class. Quant. Gravity 13, L7 (1996).

[12] M. A. Pelath and R. M. Wald, gr-qc/9901032.

[13] J. D. Bekenstein, Phys. Rev. D26, 950 (1982).

[14] J. D. Bekenstein, Phys. Rev. D27, 2262 (1983).

[15] J. D. Bekenstein, Phys. Rev. D49, 1912 (1994).

[16] J. D. Bekenstein, in Proceedings of the VIII Marcel Grossmann Meeting on General Relativity, eds. T. Piran and R. Ruffini (World Scientific, Singapore, 1999), gr-qc/9710076.

[17] J. D. Bekenstein, gr-qc/9808028.

[18] S. Hod, Phys. Rev. Letters 81, 4293 (1998) and Phys. Rev. D59, 024014 (1999).

[19] J. D. Jackson, Classical Electrodynamics (Wiley, New York, 1962).

[20] S. Hod, gr-qc/9901035.

[21] J. D. Bekenstein and A. E. Mayo, gr-qc/9903002; S. Hod, gr-qc/9903011; B. Linet, gr$\mathrm{qc} / 9903064$ and gr-qc/9904044. 